\title{
Article
}

\section{Determination of meningioma brain tumour grades using Raman microspectroscopy imaging}

Medeiros-De-morais, Camilo De lelis, Lilo, Taha Luay, Ashton, Katherine M., Davis, Charles, Dawson, Timothy P., Gurusinghe, Nihal and Martin, Francis L

Available at http://clok.uclan.ac.uk/30932/

Medeiros-De-morais, Camilo De lelis ORCID: 0000-0003-2573-787X, Lilo, Taha Luay, Ashton, Katherine M., Davis, Charles, Dawson, Timothy P., Gurusinghe, Nihal and Martin, Francis L ORCID: 0000-0001-8562-4944 (2019)

Determination of meningioma brain tumour grades using Raman microspectroscopy imaging. The Analyst, 144 (23). pp. 7024-7031. ISSN $0003-$ 2654

It is advisable to refer to the publisher's version if you intend to cite from the work. http://dx.doi.org/10.1039/c9an01551e

For more information about UCLan's research in this area go to http://www.uclan.ac.uk/researchgroups/ and search for <name of research Group>.

For information about Research generally at UCLan please go to http://www.uclan.ac.uk/research/

All outputs in CLoK are protected by Intellectual Property Rights law, including Copyright law. Copyright, IPR and Moral Rights for the works on this site are retained by the individual authors and/or other copyright owners. Terms and conditions for use of this material are defined in the policies page. 


\title{
Determination of meningioma brain tumour grades using Raman microspectroscopy imaging
}

Received 00th January 20xx, Accepted 00th January 20xx DOI: $10.1039 / \times 0 \times x 00000 x$

\author{
Camilo L. M. Morais, ${ }^{* a}$ Taha Lilo, ${ }^{a, b}$ Katherine M. Ashton, ${ }^{c}$ Charles Davis, ${ }^{a}$ Timothy P. Dawson, ${ }^{c}$ \\ Nihal Gurusinghe ${ }^{b}$ and Francis L. Martin*a
}

\begin{abstract}
Raman spectroscopy is a powerful technique used to analyse biological materials, where spectral markers such as proteins (1500-1700 cm-1), carbohydrates $\left(470-1200 \mathrm{~cm}^{-1}\right)$ and phosphate groups of DNA $\left(980,1080-1240 \mathrm{~cm}^{-1}\right)$ can be detected in a complex biological medium. Herein, Raman microspectroscopy imaging was used to investigate 90 brain tissue samples in order to differentiate meningioma Grade I and Grade II samples, which are the commonest types of brain tumour. Several classification algorithms using feature extraction and selection methods were tested, in which the best classification performances were achieved by principal component analysis-quadratic discriminant analysis (PCA-QDA) and successive projections algorithm-quadratic discriminant analysis (SPA-QDA), resulting in accuracies of $96.2 \%$, sensitivities of $85.7 \%$ and specificities of $100 \%$ using both methods. A biochemical profiling in terms of spectral markers was investigated using the difference-between-mean (DBM) spectrum, PCA loadings, SPA-QDA selected wavenumbers, and the recovered imaging profiles after multivariate curve resolution alternating least squares (MCR-ALS), where the following wavenumbers were found to be associated with class differentiation: $850 \mathrm{~cm}^{-1}$ (amino acids or polysaccharides), $1130 \mathrm{~cm}^{-1}$ (phospholipid structural changes), the region between $1230-1360 \mathrm{~cm}^{-1}$ (Amide III and $\mathrm{CH}_{2}$ deformation), $1450 \mathrm{~cm}^{-1}\left(\mathrm{CH}_{2}\right.$ bending), and $1858 \mathrm{~cm}^{-1}$ ( $\mathrm{C}=\mathrm{O}$ stretching). These findings highlight the potential of Raman microspectroscopy imaging for determination of meningioma tumour grades.
\end{abstract}

\section{Introduction}

Raman spectroscopy provides sensitive spectrochemical signatures of materials based on their molecular polarisability changes. ${ }^{1}$ Raman is based on an inelastic scattering phenomenon that occurs in less than $1 \%$ of the absorbed photons by a molecule. This inelastic scattering is composed of Stokes and anti-Stokes scattering: the former occurs when the molecule emits a photon with less energy than the absorbed incoming radiation, and the latter happens when the molecule emits a photon with higher energy than the absorbed incoming radiation. ${ }^{2}$ At room temperature, the Stokes scattering is more frequent, thus most instruments filter the elastic and anti-Stokes scattering and record the Stokes scattering signal as the final Raman spectrum.

Microspectroscopy Raman imaging allows one to obtain microscopically spatially distributed spectral data, where each position in the image is composed of a Raman spectrum in a specific

a. School of Pharmacy and Biomedical Sciences, University of Central Lancashire, Preston PR1 2HE, United Kingdom.

b. Neurosurgery, Royal Preston Hospital, Lancashire Teaching Hospitals NHS Trust, Preston PR2 9HT, United Kingdom.

c. Neuropathology, Royal Preston Hospital, Lancashire Teaching Hospitals NHS Trust, Preston PR2 9HT, United Kingdom.

*cdlmedeiros-de-morai@uclan.ac.uk/ flmartin@uclan.ac.uk

Electronic Supplementary Information (ESI) available: [details of any supplementary information available should be included here]. See DOI: $10.1039 / x 0 x x 00000 x$ wavenumber range. The hyperspectral image data are represented by three-dimensional (3D) arrays, where the spatial coordinates are present in the $x$ - and $y$-axis while the spectral information is in the $z$-axis. A major advantage of Raman imaging is that it can be nondestructive depending on the incident laser frequency, has minimum water interference, and has a relatively low cost in comparison with other analytical techniques.

Raman imaging has been used in a wide range of applications, including pharmaceutical analysis, ${ }^{3}$ forensic investigations, ${ }^{4}$ food quality control, ${ }^{5}$ and to analyse biological materials. ${ }^{6}$ In the latter, cancer detection plays an important role, where Raman imaging has been successfully applied to investigate breast, ${ }^{7}$ cervical, ${ }^{8}$ lung, ${ }^{8}$ skin, ${ }^{9}$ ovarian, ${ }^{10}$ and brain cancer. ${ }^{11}$

Most of brain cancers are gliomas or meningioma tumours. ${ }^{12}$ Gliomas are more aggressive types of tumours and have been widely investigated using Raman spectroscopy, ${ }^{12-15}$ while meningiomas remain to be intensively investigated using vibrational spectroscopy. Meningiomas represent $20 \%$ to $35 \%$ of all primary intracranial tumours. ${ }^{16}$ The majority of them occur in a supratentorial location; however, a few of them can arise in the posterior cranial fossa and, more rarely, as extracranial meningiomas. ${ }^{16}$ It usually manifests as single or sporadic lesions, causing symptoms such as sensory and motor deficits and gait disturbance; while multiple meningiomas are often associated with neurofibromatosis type 1 I. ${ }^{17}$ Meningiomas can be divided into WHO Grade I, Grade II and Grade III. Grade I meningiomas are the commonest type of tumours, with slower growth and lower 
likelihood of recurrence; Grade II meningiomas also have a slower growth but higher likelihood of recurrence; and Grade III meningiomas are a very rare type of tumour with fast growing rate and much higher likelihood of recurrence. Surgical outcomes and treatment are dependent on the meningioma grade and histological subtypes. ${ }^{17}$

In this paper, Raman microspectroscopy imaging is applied to distinguish Grade I and Grade II meningiomas via the application of several chemometric approaches, including combination of feature extraction and selection methods with discriminant analysis techniques, and multivariate curve resolution alternating least squares (MCR-ALS) for profiling and differentiation of Grade I and Grade II tumour tissues.

\section{Materials and methods}

\section{Samples}

Ninety brain tissue samples (66 meningiomas WHO Grade I, 24 meningiomas WHO Grade II) were analysed by a Renishaw InVia Basis Raman spectrometer coupled to a confocal microscope (Renishaw plc, UK). All samples were sourced from the Brain Tumour North West (BTNW) biobank (NRES14/EE/1270). All experiments were performed in accordance with the STEMH (Science, Technology, Engineering, Medicine and Health) Guidelines at the University of Central Lancashire, and approved by the ethics committee at the University of Central Lancashire (STEMH 917). Informed consents were obtained from human participants of this study. Formalin-fixed paraffin-embedded (FFPE) tissue specimens (10- $\mu \mathrm{m}$-thick) were placed onto aluminium-covered glass slides for spectroscopy measurement. Microspectroscopy imaging was performed with an acquisition area of approx. $100 \times 50 \mu \mathrm{m}(50 \times$ magnification, $785 \mathrm{~nm}$ laser, $50 \%$ laser power (150 mW), $0.1 \mathrm{~s}$ exposure time, $780-1858 \mathrm{~cm}^{-1}$ spectral range) using the StreamHRTM imaging technique (high-confocality mode) with a grid area of $42 \times 28$ pixels, resulting in 1176 spectra for each image (1 $\mathrm{cm}^{-1}$ data spacing). The laser power was set relatively high to ensure a good signal-to-noise ratio. To minimize any potential photodamage to the sample, the laser exposure time was set to only $0.1 \mathrm{~s}$. Moreover, no damage was visually observed in the samples after measurement. The imaging acquisition time was approx. $8 \mathrm{~min}$ for each sample.

\section{Computational analysis}

The Raman images were converted into suitable .txt files using the Renishaw WiRE software, and processed using MATLAB R2014b (MathWorks, Inc., USA) with lab-made routines. All the samples' images were pre-processed by cosmic rays (spikes) removal, Savitzky-Golay smoothing (window of 15 points, $2^{\text {nd }}$ order polynomial fitting), and asymmetric least squares baseline correction. The window size in the Savitzky-Golay smoothing was determined visually by testing different window sizes, where the smallest window size that removed random noise and kept the same spectral shape and intensity without smoothing-out relevant spectral peaks was chosen. MCR-ALS was applied to the image data using the HYPER-Tools toolbox in MATLAB. ${ }^{18}$

First-order classification. Each pre-processed image with size $42 \mathrm{x}$ $28 \times 1015$ was averaged into a single spectrum $(1 \times 1015)$ as the classification was performed on a sample basis. Initially, an outlier detection test was performed by a Hotelling $\mathrm{T}^{2}$ versus $\mathrm{Q}$ residuals test. ${ }^{19}$ The remaining samples after outlier removal were split into training (60\%), validation (20\%) and test $(20 \%)$ sets using the MLM sample selection algorithm. ${ }^{20,21}$ All data were mean-centred before further analysis.

For feature extraction and classification, principal component analysis combined with linear discriminant analysis (PCA-LDA), quadratic discriminant analysis (PCA-QDA) and support vector machines (PCA-SVM) were applied to the pre-processed data. PCA reduces the pre-processed spectral variables to a small number of principal components (PCs) responsible for the majority of the original data-explained variance. Each PC is orthogonal to each other and is generated in a decreasing order of explained variance, where the first PC explains most of the data variance, followed by the second PC, and so on. The PCs are composed of scores and loadings, the scores representing the variance on the sample direction, thus being used to identify similarities and dissimilarities between the samples; and, the loadings represent the variance on the wavenumber direction, being used to identify possible spectral markers associated with class differentiation. ${ }^{22}$ PCA decomposition takes the form: 22

$\mathbf{X}=\mathbf{T} \mathbf{P}^{\mathrm{T}}+\mathbf{E}$

where $\mathbf{X}$ is a matrix containing the mean-centred pre-processed spectral data; $\mathbf{T}$ is a matrix containing the PCA scores for a determined number of PCs; $\mathbf{P}$ is a matrix containing the PCA loadings for a determined number of PCs; $\mathbf{E}$ is a residual matrix; and the superscript $T$ represents the matrix transpose operation.

In PCA-LDA, PCA-QDA and PCA-SVM, a PCA model is applied to the pre-processed data and then a LDA, QDA or SVM classifier is applied to the PCA scores, respectively. LDA and QDA are discriminant analysis methods based on a Mahalanobis distance calculation. LDA assumes classes having similar variance structures, therefore using a pooled covariance matrix to calculate the classification score for each class, while QDA assumes classes having different variance structures, therefore using the variancecovariance matrix for each class individually when calculating the classification score. ${ }^{23,24}$ The LDA $\left(L_{i k}\right)$ and QDA $\left(Q_{i k}\right)$ classification scores can be calculated in a non-Bayesian form by: ${ }^{23,24}$

$L_{i k}=\left(\mathbf{x}_{i}-\overline{\mathbf{x}}_{k}\right)^{\mathrm{T}} \mathbf{C}_{\text {pooled }}^{-1}\left(\mathbf{x}_{i}-\overline{\mathbf{x}}_{k}\right)$

$Q_{i k}=\left(\mathbf{x}_{i}-\overline{\mathbf{x}}_{k}\right)^{\mathrm{T}} \mathbf{C}_{k}^{-1}\left(\mathbf{x}_{i}-\overline{\mathbf{x}}_{k}\right)$

where $\mathbf{x}_{i}$ is a vector containing the input classification variables (e.g., PCA scores) for sample $i ; \overline{\mathbf{x}}_{k}$ is the mean vector of input classification variables for class $k$; $\mathbf{C}_{\text {pooled }}$ is the pooled covariance 
matrix; and $\mathbf{C}_{k}$ is the variance-covariance matrix of class $k$. $\mathbf{C}_{\text {pooled }}$ and $\mathbf{C}_{\boldsymbol{k}}$ are calculated as follows: ${ }^{24}$

$\mathbf{C}_{\text {pooled }}=\frac{1}{n} \sum_{k=1}^{K} n_{k} \mathbf{C}_{k}$

$\mathbf{C}_{k}=\frac{1}{n_{k}-1} \sum_{i=1}^{n_{k}}\left(\mathbf{x}_{i}-\overline{\mathbf{x}}_{k}\right)\left(\mathbf{x}_{i}-\overline{\mathbf{x}}_{k}\right)^{\mathrm{T}}$

in which $n$ is the total number of samples in the training set; $K$ is the total number of classes; and $n_{k}$ is the number of samples in class $k$.

SVM is a binary linear classifier with a non-linear step called the kernel transformation. ${ }^{25} \mathrm{~A}$ kernel function transforms the input data space into a feature space by a applying a mathematical transformation which is often non-linear. Then, a linear decision boundary is fit between the closest samples to the border of each class (called support vectors), where each class is defined. SVM classification is performed as follows: ${ }^{25,26}$

$f(x)=\operatorname{sign}\left(\sum_{i=1}^{N_{S V}} \alpha_{i} y_{i} \boldsymbol{\phi}\left(\mathbf{x}_{i}, \mathbf{z}_{j}\right)+b\right)$

where $\mathbf{x}_{i}$ and $\mathbf{z}_{j}$ are vectors containing sample measurement vectors (e.g., PCA scores); $N_{S V}$ is the number of support vectors; $\boldsymbol{\alpha}_{\boldsymbol{i}}$ is the Lagrange multiplier for sample $i ; y_{i}$ is the class membership of sample $i( \pm 1) ; \boldsymbol{\phi}\left(\mathbf{x}_{i}, \mathbf{z}_{j}\right)$ is the kernel function; and $b$ is the bias parameter.

SVM was performed using a radial basis function (RBF) kernel, which is defined by: 26

$\boldsymbol{\phi}\left(\mathbf{x}_{i}, \mathbf{z}_{j}\right)=\exp \left(-\gamma\left\|\mathbf{x}_{i}-\mathbf{z}_{j}\right\|^{2}\right)$

where $\gamma$ is the kernel parameter that determines the RBF width. Cross-validation venetian blinds with 10 data splits was performed to optimise the bias and kernel parameter.

Some feature selection techniques were used to analyse the image spectral data. Successive projections algorithm (SPA) ${ }^{27}$ and genetic algorithm $(G A)^{28}$ were used coupled with LDA, QDA and SVM. SPA is a forward feature selection method which operates by minimising the co-linearity of original pre-processed spectra; thus, selecting wavenumbers whose information content is minimally redundant. ${ }^{29} \mathrm{GA}$ is an iterative algorithm inspired by Mendelian genetics, where the pre-processed spectral data is reduced to a set of selected wavenumbers based on an evolutionary process. ${ }^{28}$ For this, a set of variables is randomly chosen to go through combinations, cross-overs and mutations until the best set of variables reaches the minimum of a pre-defined cost function. ${ }^{2,28}$ The optimum number of variables for SPA and GA is obtained by minimizing the average risk $G$ of misclassification in the validation set: 29,30

$G=\frac{1}{N_{V}} \sum_{n=1}^{N_{V}} g_{n}$

where $N_{V}$ is the number of samples in the validation set and $g_{n}$ is defined by: $g_{n}=\frac{r^{2}\left(\mathrm{x}_{n}, m_{I(n)}\right)}{\min _{I(m) \neq I(n)} r^{2}\left(\mathrm{x}_{n}, m_{I(m)}\right)}$

where $r^{2}\left(\mathrm{x}_{n}, m_{I(n)}\right)$ is the squared Mahalanobis distance between sample $\mathrm{x}_{n}$ of class $I(n)$ and the centre of its true class $\left(m_{I(n)}\right)$; and $r^{2}\left(\mathrm{x}_{n}, m_{I(m)}\right)$ is the squared Mahalanobis distance between object $\mathrm{x}_{n}$ and the centre of the closest incorrect class $\left(m_{I(m)}\right)$. The GA routine was carried out using 100 generations containing 200 chromosomes each. Cross-over and mutation probabilities were set to $60 \%$ and $1 \%$, respectively. The algorithm was repeated three times, starting from different random initial populations, and the best solution in terms of fitness value was employed.

MCR-ALS. Multivariate curve resolution alternating least squares (MCR-ALS) assumes a bilinear model that is the multi-wavelength extension of the Beer-Lambert's law. It decomposes an experimental matrix $\mathbf{D}$ into concentration and spectral profiles as follows: ${ }^{31}$

$\mathbf{D}=\mathbf{C S}^{\mathrm{T}}+\mathbf{E}$

where $\mathbf{C}$ is a matrix containing the concentration profiles for a determined number of pure components in $\mathbf{D}$; $\mathbf{S}$ is a matrix containing the spectral profiles for the pure components in $\mathbf{D}$; and $\mathbf{E}$ is a residual matrix.

MCR-ALS can remove noise and physical/chemical interferences from the spectral matrix $\mathbf{D}$, and allow one to recover the pure concentration and spectral profiles of the components that make the spectral matrix D. MCR-ALS is very useful to handle image data since it allows the reconstruction of image maps based on the recovered concentration profiles, where one can identify spatial and chemical differences between the samples being imaged. ${ }^{32}$

Model validation. The models were validated by calculating some quality parameters such as accuracy, sensitivity, specificity, and Fscore. Accuracy represents the total number of samples correctly classified considering true and false negatives; sensitivity represents the proportion of positives that are correctly classified; specificity represents the proportion of negatives that are correctly classified; and, F-score measures the model performance considering imbalanced data. ${ }^{33}$ The equations to calculate these parameters are depicted in Table 1.

Table 1. Quality parameters for model validation. Where: TP stands for true positive, TN for true negative, FP for false positive, and FN for false negative.

\begin{tabular}{cc}
\hline Parameter & Equation \\
\hline Accuracy (\%) & $\left(\frac{\mathrm{TP}+\mathrm{TN}}{\mathrm{TP}+\mathrm{FP}+\mathrm{TN}+\mathrm{FN}}\right) \times 100$ \\
Sensitivity (\%) & $\left(\frac{\mathrm{TP}}{\mathrm{TP}+\mathrm{FN}}\right) \times 100$ \\
Specificity (\%) & $\left(\frac{\mathrm{TN}}{\mathrm{TN}+\mathrm{FP}}\right) \times 100$ \\
F-score & $\frac{2 \times \text { Sensitivity } \times \text { Specificity }}{\text { Sensitivity }+ \text { Specificity }}$ \\
\hline
\end{tabular}


In addition, the area under the curve (AUC) of the receiver operating characteristic (ROC) curve was evaluated to assess model quality. AUC values between 0.7 and 0.8 are considered acceptable, between 0.8 and 0.9 are considered excellent, and above 0.9 are considered outstanding. ${ }^{34}$

\section{Results and discussion}

Ninety brain tissue samples (66 meningiomas Grade I, 24 meningiomas Grade II) were analysed by Raman microspectroscopy imaging. The median microscopic and Raman image for meningiomas Grade I and Grade II are depicted in Figures 1a-1d (the colour figures represent the mean response (average Raman intensity between 780-1858 $\mathrm{cm}^{-1}$ ) of the median image for each group). Notably, each image presents different visual features due to the different distributions of chemicals on the sample surface, but their spectrochemical profile are very similar as shown in Figure $1 \mathrm{e}$ and $1 \mathrm{f}$, indicating that chemical differences between meningiomas Grade I and Grade II are not visually clear.

The pre-processed spectra from the images acquired in the

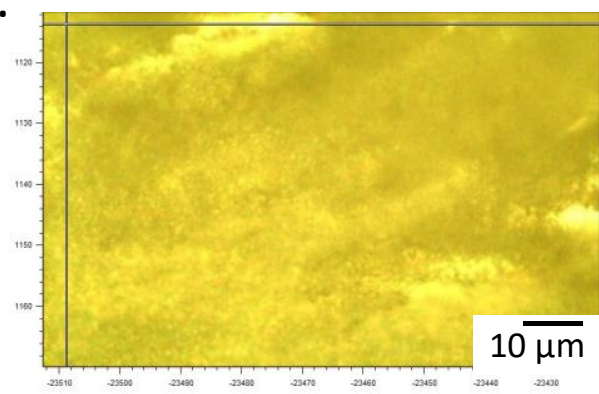

c.

Grade I

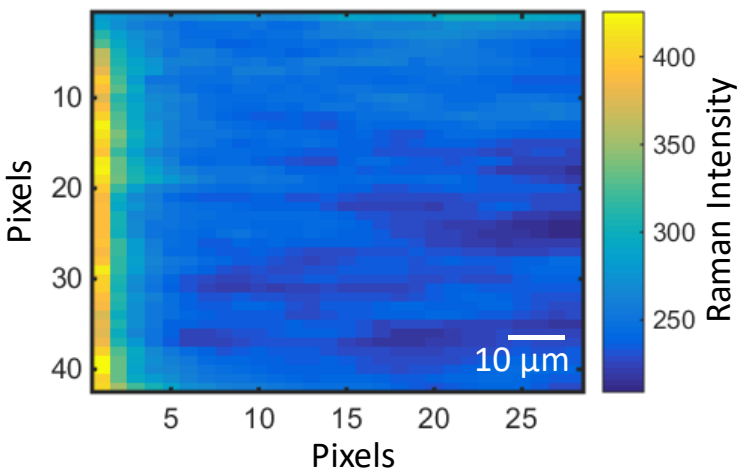

e.

Raw spectra

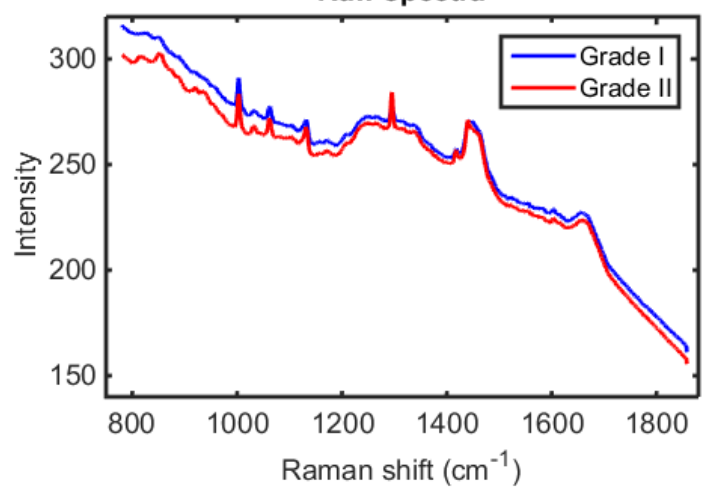

spectral range between $780-1858 \mathrm{~cm}^{-1}$ (Figure 1f) were used for further analysis. This spectral region includes the Raman fingerprint region, hence, encompassing spectrochemical signals of the main biomolecules present in the tissue samples. ${ }^{1}$ The assignment of the main peaks of the preprocessed Raman spectrum is depicted in Figure 1f. These include $\mathrm{C}-\mathrm{C}$ stretching $\left[\mathrm{v}(\mathrm{C}-\mathrm{C})_{1}\right]$ in amino acids or polysaccharides at $850 \mathrm{~cm}^{-1}, \mathrm{C}-\mathrm{C}$ stretching $\left[\mathrm{V}(\mathrm{C}-\mathrm{C})_{2}\right]$ in proteins at $890 \mathrm{~cm}^{-1}, \mathrm{C}-\mathrm{C}$ stretching $\left[\mathrm{V}(\mathrm{C}-\mathrm{C})_{3}\right]$ in amino acids at $930 \mathrm{~cm}^{-1}$, $\mathrm{C}-\mathrm{C}$ stretching $\left[v(\mathrm{C}-\mathrm{C})_{4}\right]$ in phenylalanine at $1003 \mathrm{~cm}^{-1}$, phospholipid structural changes at $1130 \mathrm{~cm}^{-1}$, Amide III peak at $1265 \mathrm{~cm}^{-1}, \mathrm{CH}_{2}$ bending $\left[\delta\left(\mathrm{CH}_{2}\right)_{1}\right]$ in lipids at $1296 \mathrm{~cm}^{-1}$, $\mathrm{CH}_{3} / \mathrm{CH}_{2}$ deformation modes in DNA/RNA at $1336 \mathrm{~cm}^{-1}, \mathrm{CH}_{2}$ bending $\left[\delta\left(\mathrm{CH}_{2}\right)_{2}\right]$ in malignant tissues at $1450 \mathrm{~cm}^{-1}, \mathrm{NH}_{2}$ bending $\left[\delta\left(\mathrm{NH}_{2}\right)\right]$ in cytosine at $1610 \mathrm{~cm}^{-1}$, and Amide I absorption at $1665 \mathrm{~cm}^{-1} .35$ Some of these peaks are discriminant features between the samples and some of them are common amongst the tumour types. The identification of relevant distinguishing spectral features between Grade I and Grade II samples are achieved by chemometric techniques.

Initially, outlier detection was performed by a Hotelling $\mathrm{T}^{2}$ versus $\mathrm{Q}$ residuals test, where 4 samples (2 meningiomas
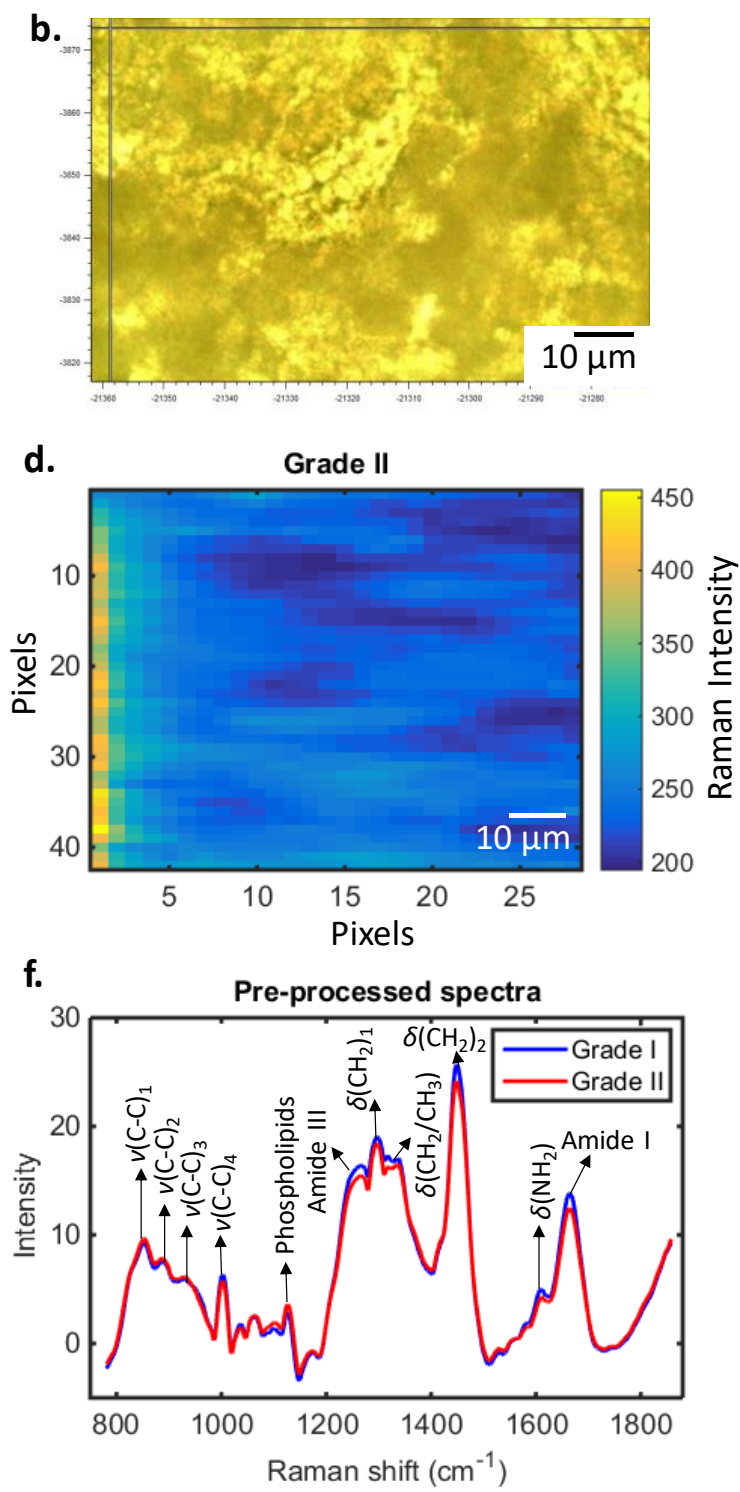

Figure 1. Median Raman miscrospectroscopy images. (a) Microscopic image of Grade I meningioma tissue; (b) microscopic image of Grade II meningioma tissue; (c) median raw image for meningioma Grade I samples; (d) median raw image for meningioma Grade II samples; (e) median raw spectra for meningiomas Grade I and Grade II; (f) median preprocessed spectra (Savitzky-Golay smoothing and asymmetric least squares baseline correction) for meningiomas with a tentative assignment of the main Raman peaks. Grade and Grade II. Colour bar: Raman intensity. $v$ : stretching vibration, $\delta$ : bending. 
Grade I, 2 meningiomas Grade II) were removed (see Electronic Supplementary Information (ESI) Figure S1). Firstorder algorithms were used to analyse the pre-processed spectral data after outlier removal.

Feature extraction and classification by means of PCA-LDA, PCA-QDA and PCA-SVM; and feature selection and classification by means of SPA-LDA, SPA-QDA, SPA-SVM, GALDA, GA-QDA and GA-SVM, were applied to distinguish meningiomas Grades I and II on sample basis. Amongst the PCA-based algorithms (using 8 PCs, 98.94\% explained variance, see ESI Figure S2), the best performance was obtained with PCA-QDA (96.2\% accuracy, $85.7 \%$ sensitivity, 100\% specificity, and F-score $=92.3 \%$ ). Also, SPA-QDA was the best algorithm amongst SPA-based methods, with the same performance of PCA-QDA. GA-based methods showed overall poorer performance, where the best algorithm (GA-QDA) achieved $73.1 \%$ accuracy but $0 \%$ sensitivity, indicating that GA-based models are most likely overfitted. More details about the predictive performance of each of these algorithms are provided in Table 2.

The ROC curve for PCA-QDA and SPA-QDA models are shown in Figure 2, where the AUC value was found at 0.929 indicating an outstanding classification performance for both algorithms.

Table 2. Quality parameter for distinguishing Grade I and Grade II meningiomas in the test set.

\begin{tabular}{lllll}
\hline Algorithm & Accuracy & Sensitivity & Specificity & F-score \\
\hline PCA-LDA & $46.2 \%$ & $85.7 \%$ & $31.6 \%$ & $46.2 \%$ \\
PCA-QDA & $96.2 \%$ & $85.7 \%$ & $100 \%$ & $92.3 \%$ \\
PCA-SVM & $61.6 \%$ & $28.6 \%$ & $73.7 \%$ & $41.2 \%$ \\
SPA-LDA & $57.7 \%$ & $100 \%$ & $42.1 \%$ & $49.3 \%$ \\
SPA-QDA & $96.2 \%$ & $85.7 \%$ & $100 \%$ & $92.3 \%$ \\
SPA-SVM & $34.6 \%$ & $71.4 \%$ & $21.1 \%$ & $32.5 \%$ \\
GA-LDA & $61.5 \%$ & $57.1 \%$ & $63.2 \%$ & $60.0 \%$ \\
GA-QDA & $73.1 \%$ & $0 \%$ & $100 \%$ & $0 \%$ \\
GA-SVM & $42.3 \%$ & $42.9 \%$ & $42.1 \%$ & $42.5 \%$ \\
\hline
\end{tabular}

QDA-based algorithms exhibit superior performance in comparison with LDA- and SVM-based methods. Usually, for complex biological data, QDA outperforms LDA since QDAbased algorithms model each class variance individually, while LDA assumes classes having similar variance structures. ${ }^{24}$ This occurs because the performance of QDA ultimately depends on the variance structure of the data. QDA is expected to work better than LDA for most biological applications, since quite commonly biological samples are composed of complex chemical matrices with different variances structures for each class. For example, diseases' samples can have a smaller variance distribution than healthy control samples, since the latter can be composed of individuals with different life habits, while patients with a same specific disease usually have a similar life-style. The same can occur with different tumour grades, where one class can assume a different variance distribution in comparison with the other. The only situation where QDA underperforms LDA is when the number of samples in the dataset is small, ${ }^{36}$ since the variance of each

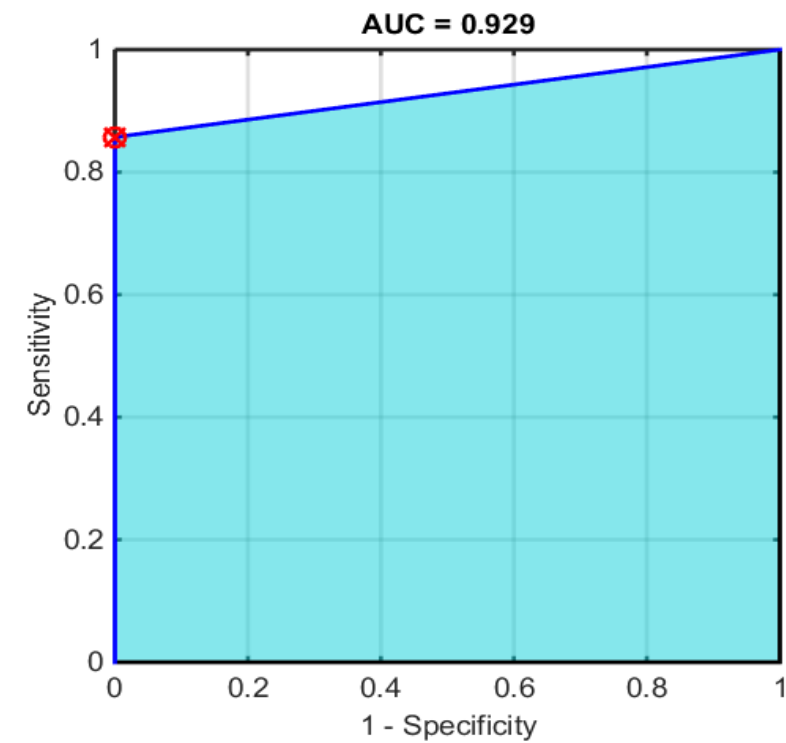

Figure 2. Receiver operating characteristic (ROC) curve for PCA-QDA and SPA-QDA AUC: area under the curve.

group might not be totally covered by QDA hence increasing the degree of extrapolation needed and commonly leading the model to overfitting.

SVM-based models seem to be highly overfitted, since the training performance for these algorithms are excellent (see ESI Table S1), with near $100 \%$ correct classification rates; however, test performance is highly affected as demonstrated in Table 2. SVM classification performance would probably improve by adding more samples to the training set, thus creating a most representative training model. Nevertheless, PCA-QDA and SPA-QDA performance are both excellent in the test set, indicating that these algorithms are robust to provide a satisfactory prediction towards external samples. 
a.
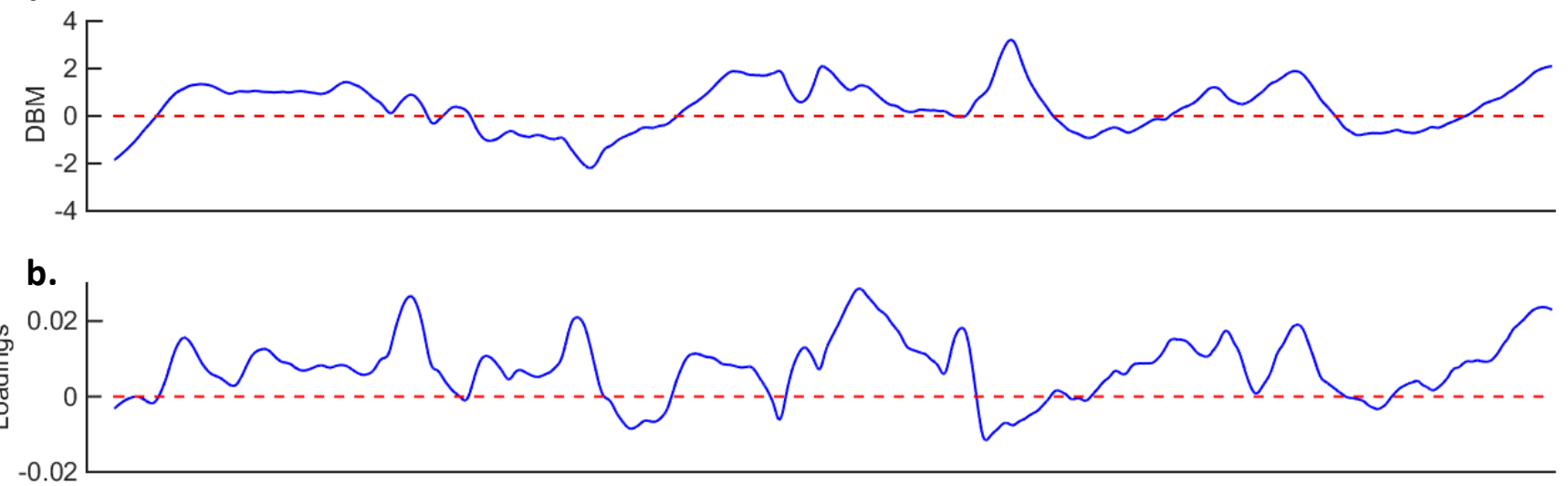

C. $\quad v(\mathrm{C}-\mathrm{C})$

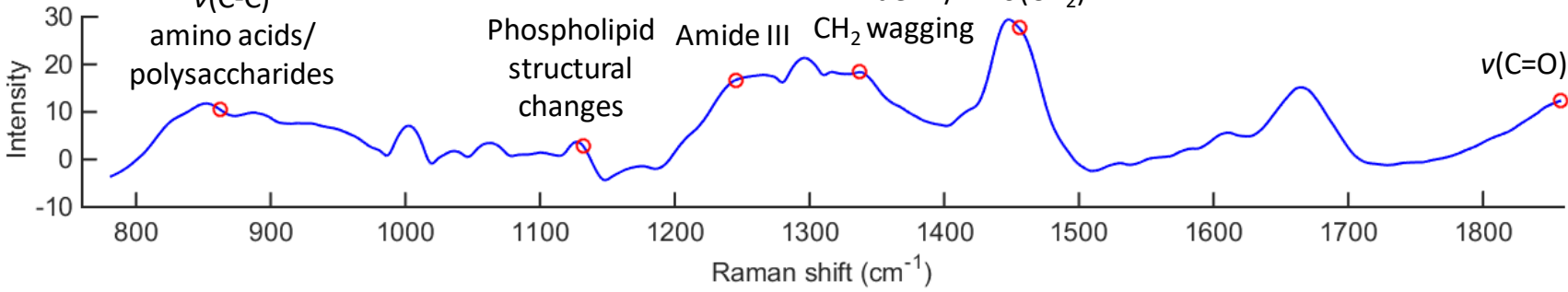

Figure 3. PCA loadings and SPA-QDA selected variables. (a) Difference-between-mean (DBM) spectrum (+ values: higher intensity in meningioma Grade I samples; - values: higher intensity in meningioma Grade II samples); (b) PCA loadings on PC1; (c) average training set spectrum and SPA-QDA selected variables (red circles) with their tentative assignment. $v$ : stretching vibration, $\delta$ : bending.

The difference-between-mean (DBM) spectrum, PCA loadings on PC1 (56.64\% explained variance), and SPA-QDA selected variables are shown in Figure 3 . The PCA loadings indicate higher coefficients at $\sim 850 \mathrm{~cm}^{-1}, \sim 1003 \mathrm{~cm}^{-1}, \sim 1130$ $\mathrm{cm}^{-1}, \sim 1337 \mathrm{~cm}-1, \sim 1450 \mathrm{~cm}^{-1}, \sim 1665 \mathrm{~cm}^{-1}$, and $\sim 1858 \mathrm{~cm}^{-1}$; and the SPA-QDA selected variables are: $\sim 850 \mathrm{~cm}^{-1}, \sim 1130 \mathrm{~cm}$ $1, \sim 1245 \mathrm{~cm}^{-1}, \sim 1337 \mathrm{~cm}^{-1}, \sim 1450 \mathrm{~cm}^{-1}$, and $\sim 1858 \mathrm{~cm}^{-1}$. Only the variable at $1245 \mathrm{~cm}^{-1}$ selected by SPA-QDA does not have a high PCA loadings, while the other variables selected by SPAQDA are very close or are a perfect match with the ones observed in PCA-QDA. The list of PCA and SPA-QDA selected variables and tentative assignment according to Movasaghi et al. ${ }^{35}$ are shown in Table 3 . The Raman shift at $1858 \mathrm{~cm}^{-1}$ is unknown based on this reference, but this wavenumber has been associated to $\mathrm{C}=\mathrm{O}$ stretching in other literature. ${ }^{37}$ The peak at around $850 \mathrm{~cm}^{-1}$ has been previously detected in meningioma samples as belonging to tyrosine, ${ }^{38}$ an $\alpha$-amino acid that constitute important structures in proteins responsible for signal transduction processes; ${ }^{39}$ and the peaks at $1003 \mathrm{~cm}^{-1}$ (phenylalanine) and $1450 \mathrm{~cm}^{-1}\left(\mathrm{CH}_{2}\right.$ bending in DNA) have also been reported as biomarkers of meningioma tumours. ${ }^{38,40}$ Phospholipids $\left(1130 \mathrm{~cm}^{-1}\right)$, Amide III $\left(1245 \mathrm{~cm}^{-1}\right)$ and Amide I $\left(1665 \mathrm{~cm}^{-1}\right)$ have been reported for brain tumours in general. ${ }^{12,40}$
Table 3. Tentative assignment of PCA and SPA-QDA selected variables to distinguish meningiomas Grade I and Grade II. DBM: difference-between-mean spectrum, where $\uparrow$ represents higher intensity in meningioma Grade I samples, and $\downarrow$ represents higher intensity in meningioma Grade II samples.

\begin{tabular}{llll}
\hline Peak & Algorithm & Assignment & DBM \\
\hline $850 \mathrm{~cm}^{-1}$ & PCA/SPA-QDA & $\begin{array}{l}\text { Amino acids or } \\
\text { polysaccharides }\end{array}$ & $\uparrow$ \\
$1003 \mathrm{~cm}^{-1}$ & PCA & $\begin{array}{l}\text { C-C in phenylalanine } \\
\text { Phospholipid }\end{array}$ & $\uparrow$ \\
$1130 \mathrm{~cm}^{-1}$ & PCA/SPA-QDA & $\begin{array}{l}\text { Phid } \\
\text { structural changes }\end{array}$ & \\
& & Amide III & $\uparrow$ \\
$1245 \mathrm{~cm}^{-1}$ & SPA-QDA & Amide III and $\mathrm{CH}_{2}$ & $\uparrow$ \\
$1337 \mathrm{~cm}^{-1}$ & PCA/SPA-QDA & wagging vibrations & \\
& & $\mathrm{CH}_{2}$ bending & $\uparrow$ \\
$1450 \mathrm{~cm}^{-1}$ & PCA/SPA-QDA & Amide I & $\uparrow$ \\
$1665 \mathrm{~cm}^{-1}$ & PCA & C=O stretching & $\uparrow$ \\
\hline
\end{tabular}

MCR-ALS was employed to resolve the median Grade I and Grade II meningioma images in order to identify spectrochemical changes associated with tumour aggressiveness. MCR-ALS was performed with 4 components selected by singular value decomposition $(99.99 \%$ explained variance, 0.21 lack of fit, non-negativity in concentration mode). The recovered concentration and spectral profiles of the 4 components are depicted in the ESI Figure S3. The $1^{\text {st }}$ component of MCR-ALS was found to be associated with Grade II appearance (Figure 4a), once it is clearly present in 
the Grade II tissue sample. The spectral profile of the $1^{\text {st }}$ component (Sopt 1) indicates distinguishing features at the region between $1230 \mathrm{~cm}^{-1}$ and $1360 \mathrm{~cm}^{-1}$ in comparison with the spectral profiles for other components (see ESI Figure S3), where three peaks $\left(1265 \mathrm{~cm}^{-1}, 1296 \mathrm{~cm}^{-1}\right.$ and $\left.1336 \mathrm{~cm}^{-1}\right)$ are presents. These peaks are associated with Amide III, $\mathrm{CH}_{2}$ deformation in lipids, and $\mathrm{CH}_{2} / \mathrm{CH}_{3}$ twisting in polynucleotide chains, respectively. ${ }^{35}$ This region encompasses the wavenumber at $1337 \mathrm{~cm}^{-1}$ (amide III and $\mathrm{CH}_{2}$ wagging vibrations) in Table 3. Similarly to Figure $1 \mathrm{f}$, the peaks at 850 $\mathrm{cm}^{-1}\left[\mathrm{v}(\mathrm{C}-\mathrm{C})_{1}\right.$, amino acids or polysaccharides], $890 \mathrm{~cm}^{-1}[\mathrm{v}(\mathrm{C}-$ C) 2 , proteins $], 930 \mathrm{~cm}^{-1}\left[\mathrm{v}(\mathrm{C}-\mathrm{C})_{3}\right.$, amino acids $], 1003 \mathrm{~cm}^{-1}[\mathrm{v}(\mathrm{C}-$ C) $)_{4}$ phenylalanine], $1130 \mathrm{~cm}^{-1}$ (phospholipids), $1265 \mathrm{~cm}^{-1}$ (Amide III), $1296 \mathrm{~cm}^{-1}\left[\delta\left(\mathrm{CH}_{2}\right)_{1}\right.$, lipids $], 1336 \mathrm{~cm}^{-1}\left[\delta\left(\mathrm{CH}_{3} / \mathrm{CH}_{2}\right)\right.$, DNA/RNA], $1450 \mathrm{~cm}^{-1}\left[\delta\left(\mathrm{CH}_{2}\right)_{2}\right.$, malignant tissue], and $1665 \mathrm{~cm}^{-}$ ${ }^{1}$ (Amide I) are also present. In addition, other peaks at 1060 $\mathrm{cm}^{-1}\left[v\left(\mathrm{PO}_{2}^{-}\right), \mathrm{DNA} / \mathrm{RNA}\right], 1100 \mathrm{~cm}^{-1}\left[\mathrm{v}(\mathrm{C}-\mathrm{C})_{5}\right.$, lipids $]$, and a small arm at $1459 \mathrm{~cm}^{-1}\left[\delta\left(\mathrm{CH}_{2}\right)_{3} \text {, deoxyribose }\right]^{35}$ are observed as distinguishing features in the MCR-ALS Sopt 1 profile.
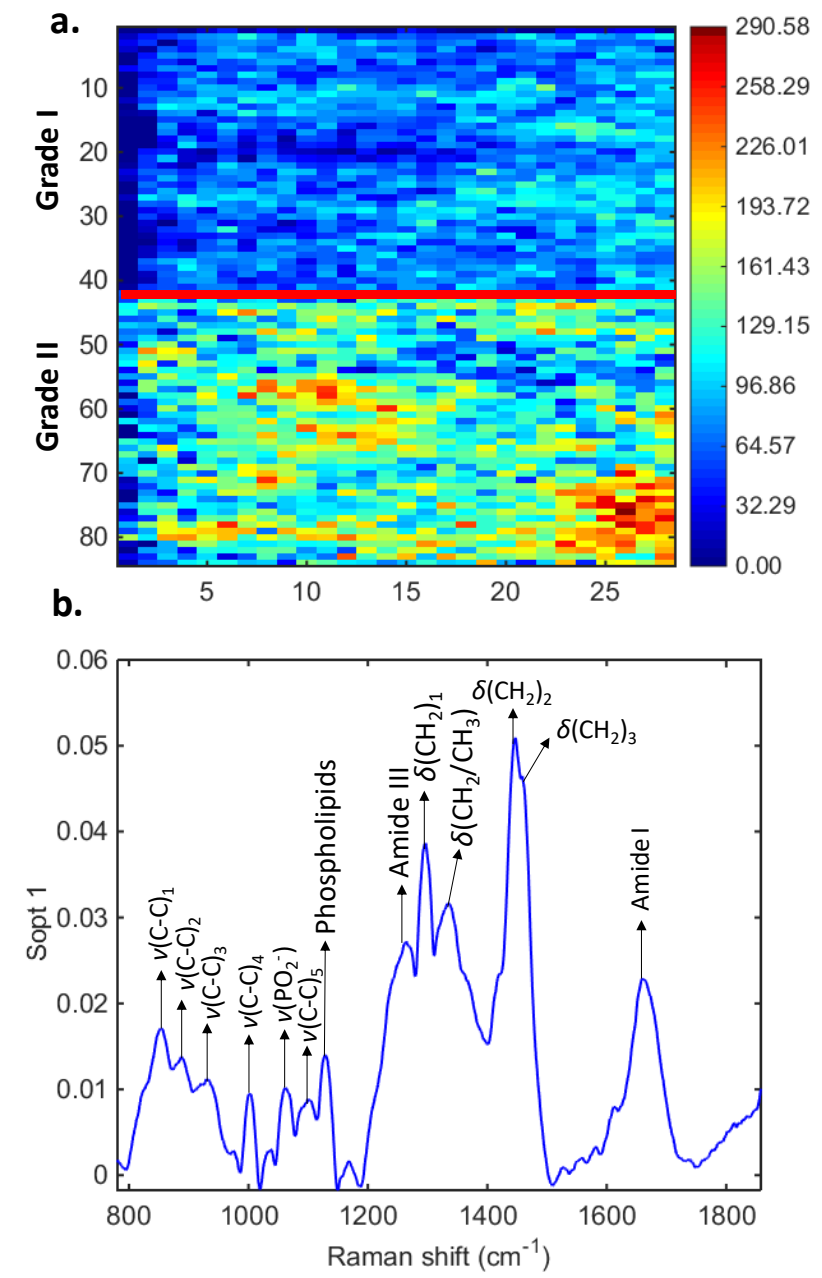

Figure 4. MCR-ALS results. (a) Recovered image using the MCR-ALS concentration profile for the $1^{\text {st }}$ component; (b) MCR-ALS spectral profile for the $1^{\text {st }}$ component with its tentative spectral markers assignment. Colour bar: relative concentration.

Bury et al. ${ }^{15}$ have recently used Raman spectroscopy to discriminate meningioma Grade I brain tissue among different brain pathologies (low-grade glioma, high-grade glioma, metastasis, lymphoma, and no-tumour) with 94.8\% accuracy, 63.9\% sensitivity and $97.1 \%$ specificity using PCA-LDA with smear-based samples; and, meningioma Grade I brain tissue among low-grade glioma, high-grade glioma, metastasis and lymphoma with $90.8 \%$ accuracy, $91.7 \%$ sensitivity and $90.8 \%$ specificity using PCA-LDA with FFPE samples. Mehta et al. ${ }^{38}$ have recently used Raman spectroscopy to discriminate healthy controls and meningioma patients based on serum using PCA-LDA. Seventy patients (35 controls, 35 meningiomas) were analysed, resulting in $70 \%$ accuracy to distinguish meningiomas versus controls in an independent test set; $72 \%$ accuracy to distinguish meningiomas Grade I versus controls; and $80 \%$ accuracy to distinguish meningiomas Grade II versus controls. The results reported herein $(96.2 \%$ accuracy, $85.7 \%$ sensitivity, $100 \%$ specificity) are very promising to distinguish meningioma tissue grades, which is critical to delineate patient treatment; and also evidences the potential of Raman spectroscopy to investigate brain tumour tissues.

\section{Conclusion}

Ninety meningioma brain tissue samples (66 meningiomas Grade I, 24 meningiomas Grade II) were investigated using Raman microscpectroscopy imaging. Several chemometric algorithms were applied to distinguish the samples according to the tumour grade, where PCA-QDA and SPA-QDA were found to have to best classification performance at $96.2 \%$ accuracy, $85.7 \%$ sensitivity and $100 \%$ specificity ( $A \cup C=0.929$ ). Spectral bio-markers at $850 \mathrm{~cm}^{-1}, 1130 \mathrm{~cm}^{-1}, 1337 \mathrm{~cm}^{-1}, 1450$ $\mathrm{cm}^{-1}$ and $1858 \mathrm{~cm}^{-1}$ were found in common using both PCAQDA and SPA-QDA, and a further analysis using MCR-ALS indicated distinguishing features at the region between $1230-$ $1360 \mathrm{~cm}^{-1}$ associated with increases in the WHO meningioma tumour grade. The classification results found by PCA-QDA and SPA-QDA are very promising, and show the potential of Raman microspectroscopy to distinguish meningioma tissue grades, thus aiding clinicians to delineate patient treatment.

\section{Conflicts of interest}

There are no conflicts to declare.

\section{Acknowledgements}

CLMM would like to thank Coordenação de Aperfeiçoamento de Pessoal de Nível Superior (CAPES) - Brazil (grant $88881.128982 / 2016-01)$ for financial support. Taha Lilo thanks Rosemere Cancer Foundation for support.

\section{References}


1 J. G. Kelly, J. Trevisan, A. D. Scott, P. L. Carmichael, H. M Pollock, P. L. Martin-Hirsch and F. L. Martin, J. Proteome Res. 2011, 10, 1437-1448.

2 M. C. D. Santos, C. L. M. Morais, Y. M. Nascimento, J. M. G. Araujo and K. M. G. Lima, Trends Anal. Chem., 2017, 97, 244256.

3 L. M. Kandpal, B. K. Cho, J. Tewari and N. Gopinathan, Sens. Actuators B Chem., 2018, 260, 213-222.

4 M. R. Almeida, L. P. L. Logrado, J. J. Zacca, D. N. Correa and R. J. Poppi, Talanta, 2017, 174, 628-632.

5 T. Yaseen, D. W. Sun and J. H. Cheng, Trends Food Sci. Technol., 2017, 62, 177-189.

6 H. J. Butler, L. Ashton, B. Bird, G. Cinque, K. Curtis, J. Dorney, K. Esmonde-White, N. J. Fullwood, B. Gardner, P. L. MartinHirsch, M. J. Walsh, M. R. McAinsh, N. Stone and F. L. Martin, Nat. Protoc., 2016, 11, 664-687.

7 H. Abramczyk and B. Brozek-Pluska, Chem. Rev., 2013, 113, 5766-5781.

8 M. Diem, A. Mazur, K. Lenau, J. Schubert, B. Bird, M. Miljković, C. Krafft and J. Popp, J. Biophotonics, 2013, 6, 855886.

9 H. Lui, J. Zhao, D. McLean and H. Zeng, Cancer Res., 2012, 72 2491-2500.

10 M. Kirsch, G. Schackert, R. Salzer and C. Krafft, Anal. Bioanal. Chem., 2010, 398, 1707-1713.

11 C. L. M. Morais, P. L. Martin-Hirsch and F. L. Martin, Analyst, 2019, 144, 2312-2319.

12 K. Gajjar, L. D. Heppenstall, W. Pang, K. M. Ashton, J. Trevisan, I. I. Patel, V. Llabjani, H. F. Stringfellow, P. L. MartinHirsch, T. Dawson and F. L. Martin, Anal. Methods, 2013, 5 , 89-102.

13 J. Desroches, M. Jermyn, M. Pinto, F. Picot, M. A. Tremblay, S. Obaid, E. Marple, K. Urmey, D. Trudel, G. Soulez, M. C. Guiot, B. C. Wilson, K. Petrecca and F. Leblond, Sci. Rep., 2018, 8, 1792.

14 L. J. Livermore, M. Isabelle, I. Bell, P. Plaha, C. Vallance and O. Ansorge, Neuro-Oncology, 2018, 20, v346.

15 D. Bury, C. L. M. Morais, K. M. Ashton, T. P. Dawson and F. L. Martin, Biosensors, 2019, 9, 49.

16 Y. Takahashi, M. Wanibuchi, Y. Kimura, Y. Akiyama, T. Mikami and N. Mikuni, World Neurosurg., 2019, 127, 525529

17 Y. Yeo, C. Park, J. W. Lee, Y. Kang, J. M. Ahn, H. S. Kang and E. Lee, Clin. Imaging, 2019, 55, 100-106.

18 N. Mobaraki and J. M. Amigo, Chemometr. Intell. Lab. Syst., 2018, 172, 174-187.

19 C. L. M. Morais, M. Paraskevaidi, L. Cui, N. J. Fullwood, M. Isabelle, K. M. G. Lima, P. L. Martin-Hirsch, H. Sreedhar, J. Trevisan, M. J. Walsh, D. Zhang, Y. G. Zhu and F. L. Martin, Nat. Protoc., 2019, 14, 1546-1577.

20 C. L. M. Morais, K. M. G. Lima and F. L. Martin, A computational protocol for sample selection in biologicalderived infrared spectroscopy datasets using Morais-LimaMartin (MLM) algorithm, Protocol Exchange, 2018. DOI: 10.1038/protex.2018.141.

21 C. L. M. Morais, M. C. D. Santos, K. M. G. Lima and F. L. Martin, Bioinformatics, 2019, btz421. DOI: 10.1093/bioinformatics/btz421.

22 R. Bro and A. K. Smilde, Anal. Methods, 2014, 6, 2812-2831.

23 S. J. Dixon and R. G. Brereton, Chemometr. Intell. Lab. Syst., 2009, 95, 1-17.

24 C. L. M. Morais and K. M. G. Lima, J. Braz. Chem. Soc., 2018, 29, 472-481.

25 C. Cortes and V. Vapnik, Mach. Learn., 1995, 20, 273-297.

26 C. L. M. Morais, F. S. L. Costa and K. M. G. Lima, Anal. Methods, 2017, 9, 2964-2970.
27 S. F. C. Soares, A. A. Gomes, M. C. U. Araujo, A. R. Galvão Filho and R. K. H. Galvão, Trends Anal. Chem., 2013, 42, 8498.

28 J. McCall, J. Comput. Appl. Math., 2005, 184, 205-222.

29 G. Theophilou, C. L. M. Morais, D. E. Halliwell, K. M. G. Lima J. Drury, P. L. Martin-Hirsch, H. F. Stringfellow, D. K. Hapangama and F. L. Martin, Anal. Bioanal. Chem., 2018, 410, 4541-4554.

30 L. F. S. Siqueira, R. F. Araújo Junior, A. A. de Araújo, C. L. M. Morais and K. M. G. Lima, Chemometr. Intell. Lab. Syst., 2017, 162, 123-129.

31 J. Jaumot, A. de Juan and R. Tauler, Chemometr. Intell. Lab. Syst., 2015, 140, 1-12.

32 J. M. Prats-Montalbán, A. de Juan and A. Ferrer, Chemometr Intell. Lab. Syst., 2011, 107, 1-23.

33 C. L. M. Morais and K. M. G. Lima, Chemometr. Intell. Lab. Syst., 2017, 170, 1-12.

34 J. N. Mandrekar, J. Thorac. Oncol., 2010, 5, 1315-1316.

35 Z. Movasaghi, S. Rehman and I. U. Rehman, Appl. Spectrosc. Rev., 2007, 42, 493-541.

36 W. Wu, Y. Mallet, B. Walczak, W. Penninckx, D. L. Massart, S Heuerding and F. Erni, Anal. Chim. Acta, 1996, 329, 257-265.

37 D. W. Mayo, F. A. Miller and R. W. Hannah, Course Notes on the Interpretation of Infrared and Raman Spectra, John Wiley \& Sons, Hoboken, 2003.

38 K. Mehta, A. Atak, A. Sahu, S. Srivastava and M. Krishna C, Analyst, 2018, 143, 1916-1923.

39 H. Kato, T. N. Faria, B. Stannard, C. T. Roberts Jr. and D. LeRoith, J. Biol. Chem., 1993, 268, 2655-2661.

40 Y. Zhou, C. Liu, Y. Sun, Y. Pu, S. Boydston-White, Y. Liu and R R. Alfano, J. Biomed. Opt., 2012, 17, 116021. 